\title{
Diagnostic promiscuity: the use of real-world data to study multimorbidity in mental health
}

\author{
Jorge Arias de la Torre, Amy Ronaldson, Jose M. Valderas, \\ Gemma Vilagut, Antoni Serrano-Blanco, Stephani L. Hatch, \\ Jordi Alonso, Matthew Hotopf and Alexandru Dregan
}

Mental health-related multimorbidity can be considered as multimorbidity in the presence of a mental disorder. Some knowledge gaps on the study of mental health-related multimorbidity were identified. These knowledge gaps could be potentially addressed with real-world data.

\section{Keywords}

Multimorbidity; mental health-related multimorbidity; real-world data; electronic health records; cohort studies.

\section{Copyright and usage}

(C) The Authors 2021. Published by Cambridge University Press on behalf of the Royal College of Psychiatrists.
Dr Jorge Arias de la Torre is a researcher at the Department of Psychological Medicine at King's College London and at the Epidemiology and Public Health Networking Biomedical Research Centre (CIBERESP), interested in multimorbidity and real-world data analysis. Dr Amy Ronaldson is a research associate working at the Department of Psychological Medicine at King's College London, looking at mental and physical multimorbidity. Professor Jose M. Valderas is a general practitioner and Professor of health services and policy at the University of Exeter Medical School, with a commitment to research on patient-centred care that is relevant for decision-making in clinical practice and health policy. Dr Gemma Vilagut is a senior statistician at the Institut Hospital del Mar Medical Research Institute, interested in data analysis related to mental health and its assessment. Dr Antoni Serrano-Blanco is Deputy Director of Mental Health at Parc Sanitari Sant Joan de Déu, and principal investigator at the Epidemiology and Public Health Networking Biomedical Research Centre (CIBERESP) and the PRISMA research group at Sant Joan de Déu Research Institute. Professor Stephani L. Hatch is a sociologist and epidemiologist leading the Health Inequalities Research Network and research programmes on inequalities in mental health and health services. Professor Jordi Alonso is Director at the Epidemiology and Public Health Programme, Hospital del Mar Medical Research Institute (IMIM), Scientific Deputy Director of Epidemiology and Public Health Networking Biomedical Research Centre (CIBERESP) and Professor at the Pompeu Fabra University. Professor Mathew Hotopf is Vice Dean of Research at the Institute of Psychiatry Psychology and Neuroscience, Professor of general hospital psychiatry at King's College London, and consultant liaison psychiatrist and Director of the National Institute of Health Research Maudsley Biomedical Research Centre at South London and Maudsley NHS Foundation Trust. Dr Alexandru Dregan is a senior lecturer in psychiatric epidemiology at the Department of Psychological Medicine at King's College London, interested in mechanisms and consequences of multimorbidity.

\section{Mental health-related multimorbidity}

Mental disorders are not only the conditions most frequently included within clusters of chronic diseases, but they may also have the largest impact on the lives of patients and their families. ${ }^{1}$ Different studies that examined combinations of chronic conditions showed that one in three patients with multimorbidity had a coexisting mental disorder. ${ }^{1,2}$ Besides, patients in clusters that contain mental disorders had worse clinical outcomes, higher premature mortality and consistently reported poorer quality of life. ${ }^{3}$ If multimorbidity could be defined as the coexistence of two or more long term or chronic diseases in one individual at a given time point, ${ }^{2,3}$ Mental health-related multimorbidity (MHRM) can be considered as multimorbidity in the presence of a mental disorder. However, despite the relevance of MHRM, its presence and rates can be particularly difficult to establish because of the overlap in symptoms between mental disorders, and the limitations of the most commonly used diagnostic classifications (mainly the DSM and ICD). ${ }^{1}$ In this sense, the promotion of a patient-centred approach and the adoption of multilayered diagnostic systems (dimensional diagnosis), such as the Research Domain Criteria or the Hierarchical Taxonomy of Psychopathology, rather than diagnostics based on specific signs and symptoms, could offer a potentially valuable approach for improving the current knowledge in MHRM.

Within the context of MHRM, as conditions may progress and become more severe, it is important to determine their trajectories and the impact on individual health outcomes during the lifespan. ${ }^{2}$ It should be noted that although the peak of onset of most of mental disorders is during adolescence and early adulthood, most of the studies on MHRM are focused on older adults. Moreover, despite the evidence about the overall health consequences of MHRM, ${ }^{1-3}$ there is a lack of actionable information that can be translated into healthcare improvements. Therefore, the study of MHRM, particularly in early stages of the lifespan, stands as a major research and clinical priority. Such research will serve to guide the development of targeted early interventions, which could help to reduce the adverse effects of MHRM over their life course.

\section{Multimorbidity and the current healthcare paradigm}

The past decades have seen an effort to enhance diagnostic precision (e.g. through the emergence of multilayered classifications in psychopathology), in an attempt to improve traditional diagnostic systems such as the DSM and ICD. This has arguably improved diagnostic accuracy and the effectiveness of treatments, allowing for the diagnosis of previously underdetected disorders (e.g. attention-deficit hyperactivity disorder in adults), the prevention and management of previously fatal diseases (e.g. certain types of cancer) and increasing the life expectancy and quality of life of patients. Paradoxically, these advances, together with the ageing of the population and its associated increase in the prevalence and incidence of chronic conditions, may have led to an increase in multimorbidity. ${ }^{2,3}$

The current healthcare paradigm favours an integrative patientcentred approach to understanding and managing complex health problems. This approach posits that the aetiology and outcomes of diseases are multifactorial and multisystemic. Multimorbidity, thus, might be attributable to a complex combination of biological factors (e.g. inflammation), behavioural factors (e.g. diet) and psychosocial factors (e.g. social deprivation). ${ }^{2,3}$ Whereas an integrative 
approach is promoted by the current healthcare paradigm, clinical practice and treatments continues to be focussing predominantly on individual conditions. In addition, there is currently no explicit approach for how to integrate the diverse, and often overlapping, clinical guidance for the management of specific physical and mental conditions. The development and promotion of evidence-based clinical guidelines focused on MHRM, which account for a wider range of determinants, might facilitate an integrative patient-centred approach.

\section{Real-world data and MHRM}

Real-world data (RWD), a popular concept in medical science in recent years, can be defined as observational data that capture healthcare outcomes in real-world settings, from heterogeneous populations and from different data sources. ${ }^{4}$ However, the type of studies included under the umbrella RWD concept can vary according to how the concept is interpreted. ${ }^{4}$ Although some interpretations encompass a broad range of study designs, including randomised controlled trials, RWD is most frequently interpreted as referring only to data from sources with inclusion criteria that are not as strict as those of clinical trials. Despite this heterogeneity, there is some consensus that two of the most relevant data sources for RWD are electronic health records (EHRs) and observational studies including cohort data. ${ }^{4}$

Rapid progress in the adoption and use of EHRs has resulted in large amounts of routinely collected clinical data becoming available for use in health research. The comprehensive nature of these data affords identification of complex patterns of multimorbidity and related burden. To date, several large data-sets comprising routine clinical data have been developed, offering an opportunity to shed light on different aspects of MHRM, ${ }^{4}$ such as the Clinical Practice Research Datalink (CPRD) in the UK, or Medicare claims data in the USA. Although comprehensive and in some cases representative, data from EHRs come with certain limitations around their completeness, accuracy and access. These data reflect clinical practice, meaning that non-clinical information, such as social determinants and life events, is often poorly captured. Similarly, mental disorders are often underdetected and underrecorded in primary care settings, a common source of EHR data in some countries, such as the UK (e.g. CPRD, QResearch databases, the Royal College of General Practitioners Research and Surveillance Centre sentinel network, and the East London Health and Care Partnership Data Repository). Furthermore, EHR data accessibility and linkage are far from straightforward processes in specific contexts, often impeded by legal and bureaucratic issues. Improving both access to EHRs, as proposed by countries such as Denmark and Sweden, and their linkage with other administrative data-sets (e.g. social care records or national insurance records) by using shared identification numbers, might be different strategies to overcome some of the current limitations. In addition, upscaling EHRs to include relevant patient-reported outcomes and risk factors could improve the quality of the data for the study of the causes and consequences of MHRM.

Within the umbrella of RWD, ${ }^{4}$ prospective epidemiological cohort studies are a complementary data source to evaluate complex processes, determinants and outcomes associated with MHRM. As strengths of cohort data, it should be mentioned that they usually include valid and reliable tools to assess different health conditions and collect detailed data on social determinants of health and patient-reported outcomes. Besides, harmonisation of measures across different cohort data enables sufficiently powered studies to identify modifiable processes underlying MHRM. ${ }^{5}$ However, cohort data also have limitations, and one of them is related to the accessibility to the samples and the possible selection bias. Patients with severe mental disorders (e.g. schizophrenia, dementia and dissociative disorders) are usually difficult to recruit in cohort studies (e.g. because of a lack of insight into their disorders or cognitive distortion and disorganisation), placing constraints on the ability of their data to identify less common (albeit clinically important) patterns of MHRM, and placing certain constraints around the external validity of their findings. Additionally, even in larger and well-supported cohort studies, the statistical power to detect MHRM patterns declines over time, because of attrition rates. To overcome these limitations, one feasible alternative could be the use of data from EHRs as they usually include entire populations continuously followed. Thus, a coordinated analysis using EHRs alongside pooled cohort data might be one approach to minimise some of their individual limitations and bring together their strengths.

\section{Opportunities and implications}

Mitigating the personal and health consequences of MHRM is contingent on elucidating the drivers of its onset, maintenance and aggravation, and the use of RWD might facilitate this. For researchers, the use of RWD could help to identify the drivers of MHRM, and further understanding of trajectories and underlying mechanisms. This could then inform the design of specific preventive interventions and treatments for clusters of pathologies that could improve the results of those treatments designed for specific diseases. For clinicians, this knowledge will serve to enhance diagnostic accuracy, improve clinical decision-making and deliver more effective and efficient treatments adapted and focused on the individual during their lifespan. Finally, for patients, using RWD in the study of MHRM could help to prevent its progression and potential consequences, enhance the results of their treatments and, consequently, improve their quality of life.

Jorge Arias de la Torre $\mathbb{D}^{\mathbb{D}}$, Institute of Psychiatry, Psychology and Neurosciences, King's College London, UK; and Epidemiology and Public Health Networking Biomedical Research Centre (CIBERESP), Spain; Amy Ronaldson, Institute of Psychiatry, Psychology and Neurosciences, King's College London, UK; Jose M. Valderas, Health Services and Policy Research Group, University of Exeter Medical School, UK; Gemma Vilagut, Health Services Research Group, Hospital del Mar Medical Research Institute (IMIM), Spain; and Epidemiology and Public Health Networking Biomedical Research Centre (CIBERESP), Spain; Antoni Serrano-Blanco $\mathbb{B}$, Epidemiology and Public Health Networking Biomedical Research Centre (CIBERESP), Spain; and Sant Joan de Déu Research Institute, Parc Sanitari Sant Joan de Déu, Spain; Stephani L. Hatch, Institute of Psychiatry,

Psychology and Neurosciences, King's College London, UK; and ESRC Centre for Society Psychology and Neurosciences, King's College London, UK; and ESRC Centre for Society
and Mental Health, King's College London, UK; Jordi Alonso $\mathbb{D}$, Hospital del Mar Medical Research Institute (IMIM), Spain; and Epidemiology and Public Health Networking Biomedical Research Centre (CIBERESP), Spain; Matthew Hotopf $\mathbb{D}$, Institute of Psychiatry, Psychology and Neurosciences, King's College London, UK; and South London and Maudsley NHS Foundation Trust, UK; Alexandru Dregan (D), Institute of Psychiatry, Psychology and Neurosciences, King's College London, UK

Correspondence: Jorge Arias de la Torre. Email: jorge.arias de_la_torre@kcl.ac.uk

First received 17 Sep 2020, final revision 23 Oct 2020, accepted 9 Dec 2020

\section{Author contributions}

All authors (J.A.T., A.D., A.R., J.M.V., G.V., A.S.B., S.L.H., J.A. and M.H.) contributed equally to the conception of this work. J.A.T and A.D. drafted the article, and A.R., J.M.V., G.V., A.S.B., S.L.H., J.A. and M.H. provided critical revision.

\section{Funding}

The present work is funded by the Medical Research Council (grant number MR/S028188/1). This article represents independent research part funded by the National Institute for Health Research (NIHR) Biomedical Research Centre at South London and Maudsley NHS Foundation Trust and King's College London. The views expressed are those of the authors and not necessarily those of the NHS, the NIHR or the Department of Health.

\section{Declaration of interest}

M.H. is principal investigator of the RADAR-CNS programme, a precompetitive public-private partnership funded by the Innovative Medicines Initiative and European Federation of Pharmaceutical 
Industries and Associations. The programme receives support from Janssen, Biogen, MSD, UCB and Lundbeck. All other authors declare that they have no competing interests.

ICMJE forms are in the supplementary material, available online at https://doi.org/10.1192/ bip 2020.257

\section{References}

1 Langan J, Stewart WM, Smith DJ. Multimorbidity and mental health: can psychiatry rise to the challenge? Br J Psychiatry 2013; 202(6): 391-3.
2 Barnett K, Mercer SW, Norbury M, Watt G, Wyke S, Guthrie B. Epidemiology of multimorbidity and implications for health care, research, and medical education: a cross-sectional study. Lancet 2012; 380: 37-43.

3 Whitty CJM, MacEwen C, Goddard A, Alderson D, Marshall M, Calderwood C, et al. Rising to the challenge of multimorbidity. BMJ 2020; 368: 16964

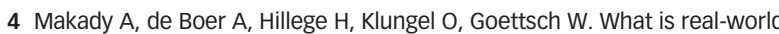
data? A review of definitions based on literature and stakeholder interviews. Value Heal 2017; 20(7): 858-65.

5 Hutchinson DM, Silins E, Mattick RP, Patton GC, Fergusson DM, Hayatbakhsh R, et al. How can data harmonisation benefit mental health research? An example of the Cannabis Cohorts Research Consortium. Aust N Z J Psychiatry 2015; 49(4): 317-23.

\section{0 words}

\section{0 words on lateral inhibition}

Jakov K. Zlodre (iD

Lateral inhibition is a neurobiological mechanism whereby the boundaries of stimuli are enhanced in the retina and peripheral nervous system, such as touch in the skin. Dendritic trees and interneuron populations, which mediate a mechanism similar to lateral inhibition within the cerebral cortex, are reduced in people with a history of psychosis. The loss of cortical surround inhibition could result in a loss of 'cognitive definition', a loss of gain on signalling as it passes from stimulus to perception, memory and action, leading to loosening of associations, reality distortion such as hallucinations and delusions and disorganisation of thought and behaviour.

(c) The Author(s), 2021. Published by Cambridge University Press on behalf of the Royal College of Psychiatrists 